\title{
Editorial commentary on the Indian Journal of Gastroenterology January-February 2021
}

\author{
Jimmy K. Limdi ${ }^{1,2,3}$ (D) \\ Published online: 5 March 2021 \\ (C) Indian Society of Gastroenterology 2021
}

\section{Idiopathic acute pancreatitis-A myth or reality? Role of endoscopic ultrasonography and magnetic resonance cholangiopancreatography in its diagnosis}

Modern diagnostic modalities have enhanced the ability to diagnose many conditions that may previously have been labeled as "idiopathic". Between $10 \%$ to $30 \%$ of patients with acute pancreatitis in whom standard investigations did not reveal etiology have been labeled as having "idiopathic acute pancreatitis" (IAP) [1]. In a prospective study of 31 patients with "IAP", Mitra et al. from Sir Sunderlal Hospital, Institute of Medical Sciences, Banaras Hindu University, Varanasi, India evaluated the ability of modern hepatobiliary investigations in elucidating an etiology [2]. Endoscopic ultrasound (EUS) and/or magnetic resonance cholangiopancreatography (MRCP), performed at least 4 weeks after a diagnosis of IAP. EUS established a diagnosis in $14(45.2 \%)$ patients. The diagnoses were microlithiasis, gallbladder sludge, gallstones, pancreatic adenocarcinoma and intraductal papillary mucinous neoplasm (IPMN). MRCP enabled a diagnosis in 8 $(25.8 \%)$ patients, demonstrating microlithiasis, gallstones, pancreatobiliary anomalies, pancreatic adenocarcinoma and IPMN. EUS diagnosed more etiologies in patients without previous cholecystectomy. Based on these observations, EUS may be preferred for the identification of biliary etiology and MRCP for anatomical pancreatobiliary pathology causing IAP.

Jimmy K. Limdi

Jimmy.Limdi@nhs.net

1 Division of Gastroenterology, The Pennine Acute Hospitals NHS Trust, Manchester, UK

2 Manchester Academic Health Sciences, University of Manchester, Manchester, UK

3 Manchester Metropolitan University, Manchester, UK

\section{Prognostic impact of incidentally detected hepatocellular carcinoma in explanted livers after living donor liver transplantation}

There is considerable controversy around the impact of incidentally detected hepatocellular carcinoma (iHCC) in explanted livers on the prognosis of patients undergoing orthotopic liver transplantation. Rajakannu et al. from Dr. Rela Institute and Medical Centre, Chennai, India, and Bharat Institute of Higher Education and Research, Chennai, India describe a retrospective experience from 545 adults undergoing living donor liver transplantation (LDLT) over a 9year period [3]. They detected iHCC in 28 patients (5.1\%), with no recurrence over a median follow-up of 28 months. The 5-year overall and recurrence-free survival was also encouraging at $96.4 \%$, leading the authors to conclude that specific post-transplant surveillance or treatment is probably unnecessary. It is noteworthy, that pre-operative screening for LDLT is rigorous, with patients undergoing high-quality triple-phase CT and/or MRI with diffusion weighted imaging with high sensitivity for even small lesions. Furthermore, the duration between listing and LDLT is very short (4-6 weeks), making it unlikely that small and indeterminate lesions would progress. Studies reporting longer follow-up will be informative.

\section{Argon plasma coagulation - An effective treatment for solitary rectal ulcer syndrome: A single-center experience from western India}

Solitary rectal ulcer syndrome (SRUS) is a benign, uncommon and likely underreported disorder of defecation affecting approximately 1 in 100,000 people per year [4]. It is characterized by symptoms of bloody discharge associated with mucus and a sense of incomplete evacuation. Conventional therapies such as laxatives, biofeedback and topical treatments are often ineffective. Consequently, SRUS is associated with 
considerable morbidity with a poor quality of life. Some patients may require surgical treatments. Shah and colleagues from Apollo Hospitals International Limited, Gandhinagar, India report a retrospective experience of using argon plasma coagulation (APC) in 14 patients with refractory SRUS [5]. Patients required between 1 and 5 APC sessions after which non-healing was deemed as treatment failure. Control of bleeding was achieved in $100 \%$ patients, complete ulcer healing in $71 \%$ and $>50 \%$ reduction in ulcer size in $29 \%$ of patients with no procedure related complications over a mean follow-up period of 7 months. Larger multicenter studies with longer follow may validate these findings.

\section{Efficacy of combining Pentoxiphylline and vitamin $E$ versus vitamin $E$ alone in non-alcoholic steatohepatitis - A randomized pilot study}

Non-alcoholic fatty liver disease (NAFLD) is the most common cause of chronic liver disease, an important cause of hepatocellular carcinoma and is expected to become the leading cause of liver transplantation by 2030 [6]. Abrogation of the necro-inflammatory state (non-alcoholic steatohepatitis or NASH) may retard early fibrosis and further progression of liver injury. Kedarisetty and colleagues, from Institute of Liver and Biliary Sciences, New Delhi, India report a prospective study of histologically proven patients with NASH randomized to receive either vitamin E (VE) $400 \mathrm{IU}$ bd alone $(n=33)$ or VE combined with pentoxiphylline (PTX) $400 \mathrm{mg}$ tds $(n=36)$ (PTVE group) [7]. Both groups adhered to a programme of strict diet and lifestyle modification. The primary endpoint of "reduction in alanine aminotransferase (ALT) levels" was similar in both groups, although the PTVE group had greater reductions in insulin and tumor necrosis factor alpha $(\mathrm{TNF} \alpha)$ from 6 months onwards. Although VE was noted to be as effective as combination of PTX and $\mathrm{VE}$, further controlled studies assessing this combination on biochemical, cytokine and histological parameters are needed.

\section{Gastric secretion in patients with caustic ingestion: A prospective study}

Caustic ingestion with suicidal intent tragically remains relatively common from unregulated access to over-the counter acid substances. Although immediate complications such as vomiting, hematemesis and late stricture formation are known, less is known about basal and stimulated gastric acid output and its pathophysiological consequences [8]. Shah and colleagues from Postgraduate Institute of Medical Education and Research, Chandigarh, India studied basal and pentagastrin stimulated gastric secretion in 16 patients with a history of caustic ingestion and noted significantly lower mean gastric acid secretion compared to controls [9]. Mean gastric juice volume and acidity increased after pentagastrin stimulation but was lower than in controls. Patients with a lower esophageal stricture had decreased maximum acid output and consequently higher risk of hypochlorhydria compared to patients with stricture in the upper or middle esophagus. Hypochlorhydria increases the risk of vitamin $\mathrm{B}_{12}$, iron and calcium deficiency as also the risk of bacterial overgrowth and carcinoma. Consequently, patients with caustic esophageal and antral narrowing with hypochlorhydria do not need acid-suppressant treatment as is given with peptic strictures. Further mechanistic studies are needed.

\section{Prevalence and risk factors for gastroesophageal reflux disease in a rural Indian population}

Although a substantial proportion (13.98\%) of the global population suffers from gastroesophageal disease (GERD), significant variations exist between regions and countries and data from Asia and particularly from rural areas are scarce [10]. Ghoshal and colleagues from Sanjay Gandhi Postgraduate Institute of Medical Sciences, Lucknow, India report a large survey from 4 villages in northern India with the aim of assessing frequency and risk factors associated with GERD [11]. This household survey administered a translated and validated version of the Enhanced Asian Rome III and Hospital Anxiety and Depression questionnaire to 2774 individuals. Of these, $27.2 \%$ reported heartburn, with $11.3 \%$, $5.2 \%$ and $3.1 \%$ reporting the frequency as 1,2 and 3 times a week. GERD was reported in $10.7 \%$ of the surveyed population. Factors associated with GERD were elevated BMI $>25 \mathrm{~kg} / \mathrm{m}^{2}$, predominant rice eating, tobacco chewing, smoking, alcohol and intake of carbonated drinks. A high proportion (41\%) of these also reported functional dyspepsia and indeed psychological comorbidities were more common in those with GERD than in those without.

\section{A novel homozygous frameshift variant in the ABCC2-gene in Dubin-Johnson syndrome may predispose to chronic liver disease}

Dubin-Johnson syndrome (DJS) is a rare autosomal recessive that usually presents in the second decade of life with conjugated hyperbilirubinemia. Pathogenic variants in the $\mathrm{ABCC} 2$ gene are implicated. The condition is benign and not believed to progress to chronic liver disease. Philips and colleagues from Cochin Gastroenterology Group, Ernakulam Medical Center, Kochi, India report a novel frameshift sequence 
variant in the $\mathrm{ABCC} 2$-gene in 3 family members of a family affected by DJS [12]. This variant, c.4406 4407delTA (p.Leu1469fs) was associated with chronic liver disease in the absence of any other attributable etiology and evidence of extensive hepatocellular melanin-like pigment staining on the background of cirrhosis. Larger population based and functional studies are needed to validate this novel finding and its association with chronicity of liver disease.

\section{Monitoring photographic proof of cecal intubation: A closed-loop audit of best practice colonoscopy}

Colonoscopy remains the gold-standard for colonic investigation and diagnosis of dysplasia, polyps and colorectal cancer as also inflammatory bowel disease. Cecal intubation is one of many key performance indices used to document complete colonic evaluation. International societies advocate photographic evidence of cecal landmarks as evidence of cecal intubation [13].

Whitehead-Clarke and colleagues from the University College. London, UK, and Epsom and St Helier NHS Trust, UK present an audit on compliance with this standard from their institution [14]. Of 200 consecutive colonoscopies assessed in the first audit, $70 \%$ provided photodocumentation of the cecum. Following presentation of findings and guidelines, a subsequent audit failed to demonstrate $100 \%$ compliance, with $71 \%$ of procedures providing satisfactory proof of cecal intubation. Successful colonoscopy hinges on achieving and maintaining high standards with key performance indices, of which accurate cecal intubation with photo-documentation is a key aspect for patient safety, competency assessment and indeed for medico-legal reasons and must be implemented by all gastroenterologists performing colonoscopy.

\section{Ischemic jejunal stricture in patients with extrahepatic portal vein obstruction}

Patient with extrahepatic portal vein obstruction (EHPVO) typically have a benign course although it may be complicated by hypersplenism, variceal bleeding and portal biliopathy [15]. Ischemic jejunal stricture from mesenteric vein thrombosis (MVT) is a rare complication of EHPVO and can present with small bowel obstruction. Priyadarshi and colleagues from All India Institute of Medical Sciences, Patna, India report 3 cases of jejunal strictures as a complication of MVT and describe the diagnosis, work-up and computed tomography (CT) imaging findings [16]. Two of the 3 patients underwent surgical resection and there was one death in a patient deemed unfit for surgery. Although routine use of anticoagulation in
EHVPO is still debated, long-term anticoagulation is recommended in those with a documented prothrombotic state [15].

\section{Compliance with ethical standards}

Conflict of interest JKL declares that he has no conflict of interest.

Disclaimer The author is solely responsible for the data and the contents of the paper. In no way, the Honorary Editor-in-Chief, Editorial Board Members, the Indian Society of Gastroenterology, or the printer/ publishers are responsible for the results/findings and content of this article.

\section{References}

1. Guda NM, Trikudanathan G, Freeman ML. Idiopathic recurrent acute pancreatitis. Lancet Gastroenterol Hepatol. 2018;3:720-8.

2. Mitra T, Dixit VK, Yadav DP, et al. Idiopathic acute pancreatitisA myth or reality? Role of endoscopic ultrasonography and magnetic resonance cholangiopancreatography in its diagnosis. Indian J Gastroenterol. 2021;40. https://doi.org/10.1007/s12664-02001125-9.

3. Rajakannu M, Vij M, Shaikh TMA, Rammohan A, Reddy MS, Rela M. Prognostic impact of incidentally detected hepatocellular carcinoma in explanted livers after living donor liver transplantation. Indian J Gastroenterol. 2021;40.https://doi.org/10.1007/ s12664-020-01127-7.

4. Sharma A, Misra A, Ghoshal UC. Fecal evacuation disorder among patients with solitary rectal ulcer syndrome: a case-control study. J Neurogastroenterol Motil. 2014;20:531-8.

5. Shah A, Bohra S, Desai S. Argon plasma coagulation - an effective treatment for solitary rectal ulcer syndrome: A single-center experience from western India Indian J Gastroenterol. 2021;40. https:// doi.org/10.1007/s12664-020-01089-w.

6. Chalasani N, Younossi Z, Lavine JE, et al. The diagnosis and management of nonalcoholic fatty liver disease: practice guidance from the American Association for the Study of Liver Diseases. Hepatology. 2018;67:328-57.

7. Kedarisetty CK, Bhardwaj A, Rastogi A, et al. Efficacy of combining Pentoxiphylline and Vitamin E versus Vitamin E alone in nonalcoholic steatohepatitis - A randomized pilot study. Indian J Gastroenterol, 2021; 40. https://doi.org/10.1007/s12664-02001131-x.

8. Chirica M, Bonavina L, Kelly MD, et al. Caustic ingestion. Lancet. 2017;389:2041-52.

9. Shah J, Bush N, Rajan K, et al. Gastric secretion in patients with caustic ingestion: A prospective study. Indian J Gastroenterol. 2021;40. https://doi.org/10.1007/s12664-020-01116-w.

10. Nirwan JS, Hasan SS, Babar ZU, et al. Global prevalence and risk factors of gastro-oesophageal reflux disease (GORD): systematic review with metaanalysis. Sci Rep. 2020;10:5814.

11. Ghoshal UC, Singh R, Rai S. Prevalence and risk factors of gastroesophageal reflux disease in a rural Indian population. Indian $\mathrm{J}$ Gastroenterol. 2021;40.https://doi.org/10.1007/s12664-02001135-7.

12. Philips CA, Agarwal M, Rajesh S, et al. A novel homozygous frameshift variant in the ABCC2-gene in Dubin-Johnson syndrome may predispose to chronic liver disease. Indian J Gastroenterol. 2021;40.https://doi.org/10.1007/s12664-020-01121-z.

13. Rees CJ, Thomas Gibson S, Rutter MD, et al. UK key performance indicators and quality assurance standards for colonoscopy. Gut. 2016;65:1923-9. 
14. Whitehead-Clarke T, Sothinathan R, Sandhya MA, et al. Monitoring photographic proof of cecal intubation: A closed-loop audit of best practice colonoscopy. Indian J Gastroenterol. 2021;40. https://doi.org/10.1007/s12664-020-01085-0.

15. Kumar A, Sharma P, Arora A. Review article: portal vein obstruction-epidemiology, pathogenesis, natural history, prognosis and treatment. Aliment Pharmacol Ther. 2015;41:276-92.
16. Priyadarshi RN, Anand U, Kumar R, et al. Ischemic jejunal stricture in patients with extrahepatic portal vein obstruction. Indian J Gastroenterol. 2021;40.https://doi.org/10.1007/s12664-02001123-x.

Publisher's note Springer Nature remains neutral with regard to jurisdictional claims in published maps and institutional affiliations. 